\title{
Bridging Multi-light \& Multi-Spectral images to study, preserve and disseminate archival documents
}

\author{
Bruno Vandermeulen; LS Digitisation and Document Delivery, KU Leuven, Leuven, Belgium \\ Hendrik Hameeuw; LS Digitisation and Document Delivery, KU Leuven, Leuven, Belgium \\ Lieve Watteeuw; Faculty of Theology and Religious Studies, Illuminare, Book Heritage Lab, KU Leuven, Leuven, Belgium \\ Luc Van Gool; ESAT, KU Leuven, Leuven, Belgium \\ Marc Proesmans; ESAT, KU Leuven, Leuven, Belgium
}

\begin{abstract}
Producing relevant photographic records in collections with unique and often fragile heritage objects is a serious challenge. Combining both visual and analytic information into such images is a major asset. Over the last decade the development of the Leuven University Portable Light Dome (PLD) has produced numerous such complex datasets. Its outcome enables different visualizations and analyses on one and the same multi-light and/or multi-spectral dataset. These interactive images support various types of research questions and contain many facets of information (reflectance characteristics, surface orientations, multi-spectral). Compared to normal photography they contain much more layered information on the archived objects. Compared to other multi-light reflectance imaging solutions such as RTI, the imaging protocol of the PLD system is able to disseminate its outcome in a multi-modal manner, beyond the visual aspects of the imaged surface.
\end{abstract}

\section{Developing a suitable acquisition and dissemination method: The WL and MS Portable Light Dome Systems}

As the imaging-needs and demands of curators and researchers can differ, and in particular cases even conflict, several types of visual representations of heritage objects are produced. Therefore, the more information one imaging-routine can record the more diverse questions can be met; ideally providing data sets allowing interactive engagement with the object and thus opening a spectrum of potential research- or conservation-driven actions.

The photographic records and data sets created with the Portable Light Dome (PLD) system, developed at the KU Leuven, provide a suitable strategy to satisfy these needs of researchers, curators and conservation scientists. Since 15 years the PLD system was used for the digitization and imaging of large, often isolated and very diverse heritage collections. The White Light (WL) PLD acquisition system was initially developed for the accurate reconstruction of the surface orientation of 3rd to 1 st millennium BCE cuneiform archives and seal impressions. The viewer, with its custom-made and fine-tuned shaders, provided results supporting research; allowing genuine digital preservation of these extreme fragile artifacts and facilitated the publication of the content through the integrated automated visualization options [1][2][3]. By 2012, within the RICH project, the WL PLD hardware was adapted and miniaturized for the safe monitoring of documentary heritage objects [4][5][6][7]. These new developments enabled high-end, high-resolution analyses of the topography and materiality of book, paper and parchment heritage objects, all based on the reflective characteristics and estimated surface orientations, inherent to principles of photometric stereo applied on the acquired data sets with the multi-light reflectance system of the PLD (for results see also infra).

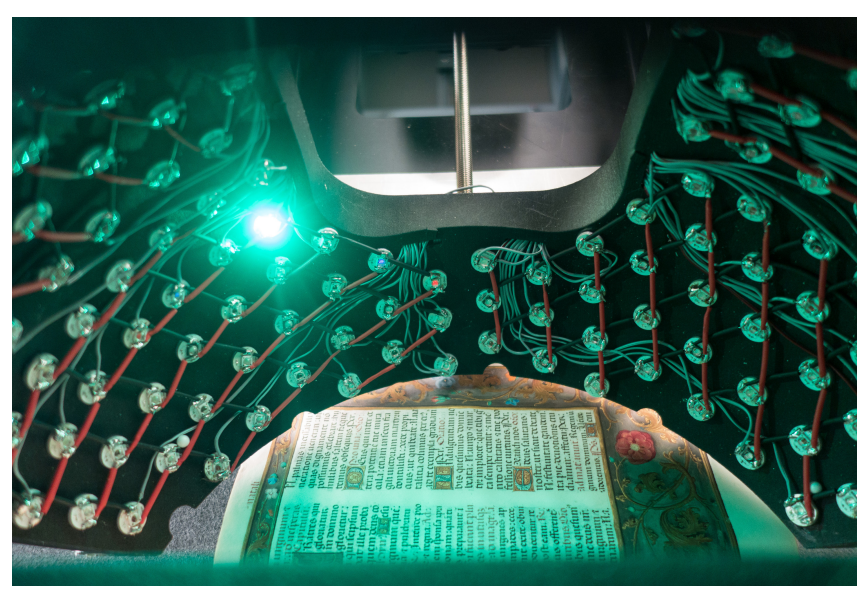

Figure 1. The MS PLD system recording an illuminated manuscript, with one of the Green 523nm LEDs in action (@ Imaging Lab - KU Leuven).

Joining the efforts in 2014, the RICH and EES projects opened up new paths for the research with this technology. Within this framework multi-light reflectance and multi-spectral imaging techniques were merged by integrating 5 different spectral bands in the acquisition dome. This has increased individually and conjointly the possible interpretation models vastly [8][9]. Parallel work has demonstrated the potential of this approach [10][11] and since then a number of case studies powered with by RTI tools on archaeological artifacts [12], painted surfaces [13] and papyri [14] have been published. This MS PLD system (Figure 1) enables the study of the imaged surfaces from multiple perspectives. The MS reflective responses of each registered point (pixel) can be compared 1:1 with surface orientations calculated through distinct data sets derived from IR $(850 \mathrm{~nm})$, Red $(623 \mathrm{~nm})$, Green $(523 \mathrm{~nm})$, Blue (460) and UV (365nm) spectral bands. This approach enables the measurement in three dimensions of the topography of objects in a $2 \mathrm{D}+$ environment (Figure 2). In addition to that, the same (MS) datasets allow the photometric stereo algorithm with the reflectance response estimations for every recorded pixel analyzing the materiality of the imaged surface [9].

Throughout the development phases of the different PLD acquisition modules and software solutions the strategy remained that the entire registration and processing routine needed to be as easy as possible, where a good and balanced time / quality ratio was pursued. An important incentive to achieve this goal is the automated all-in-one approach; once the surface has been 
positioned in line with the acquisition dome, one simple recording sequence orderly produces all the needed data for the processing and eventual consultation. Due to this fact, the recording sequence can be easily replicated e.g. after conservation allowing in depth comparison of the different physical states of the object.

\section{Demands of the Field}

The balanced development of the acquisition and dissemination tools is challenging, as the demands formulated by conservation and collection care, researchers and students don't always conjure to the research questions addressed or raised by the engineers. Stimulatingly, the bonding factor fusing interdisciplinary collaboration between engineers, conservators, researchers, imaging technicians and curators is the common sense and mutual understanding that care, knowledge, access and preservation of cultural heritage objects is vital.

With the PLD system, divergent categories of heritage objects have already been monitored such as charters, legal documents, manuscripts, sewing threads, drawings, prints, seals, stamps, watermarks, papyri, paintings, coins and many types of archaeological objects. Close interaction between various researchers acquainted with these different artifact categories, hard- \& software developers, image technicians and collection care have each time formed the base of the research projects that steered the aforementioned developments of the PLD system. This has led to a wide array of strategies to monitor, study and disseminate these various artifact categories. Thanks to this close collaboration with all involved partners, both visual and analytical/metric data could be incorporated and combined to enhance the understanding of the original object, all based on one single recording sequence.

\section{Dissemination strategies}

All raw data is archived and can be reprocessed in the case future features in the software are developed. A layered solution is implemented to access and interact with these files (.cun $\&$.zun). The native desktop viewer (by now PLDviewer version 7.0.04/05) allows both visual and analytic assessment; it exploits all the power of your graphics card for real-time, high-resolution viewing and feedback, and provides tools for live calculation and visualization of metric data such as the measurement tool (X, Y and $\mathrm{Z}$ axis), multi-spectral histograms and BRDF projections.

The online viewer (www.minidome.be) on the other hand focuses on the broader dissemination and the fast browsing of the available digitized archival documents. It uses WebGL as a backbone for direct interactive viewing in a browser. Although it is more limited in terms of memory and computational resources, due to the fact that there is no need for installing dedicated plugins, it gives an instant feel and look on the underlying data. The software architecture of the online viewer allows consulting digitized content both via links incorporated in webpages and databases or by direct upload of any local stored data set. Future work (the Pixel+ project) will focus on merging the technologies of the PLD system (.cun \& .zun) and the RTI system (.ptm \& .rti) by developing a Windows/Mac OS X/Linux viewer and a web viewer, which will be capable in displaying all these file formats with their respective shaders and metadata.

Various data-formats can be exported ranging from low and full resolution image export to metric data allowing further calculation and visualization. All working data is managed by a digital asset management system linking interactive files with the various derivative exports.

\section{Selection of results with parchment \& paper media}

(sub-)Project by (sub-)project the needs for the study, digital preservation and dissemination of various object categories have been tackled. Each time the multi-light/multi-spectral reflectance technology of the PLD system was tested with the new types of objects and materials, in particular defined by their materiality, shape and dimensions. In-depth visualizations through advanced filters, extraction of metric data, metric and visual monitoring for conservation, identification of materials and allowing comparison of material characteristics on the topography of heritage objects has provided a set of strategies all incorporated through the aforementioned native desktop viewer for the PLD system. Below we will only focus on examples obtained with parchment and paper media.

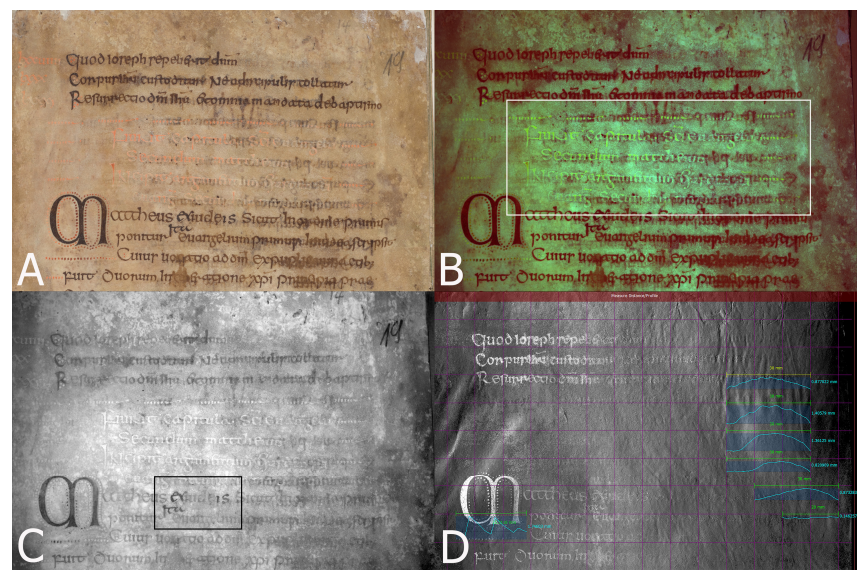

Figure 2. Detail 01 on folio 19v of the 8th century Codex Eyckensis, parchment (@ Musea Maaseik) - (A) Phase One RGB image acquisition according to Metamorfoze standard; $(B-D)$ Renderings based on one and the same 5 min recording with the MS PLD system; (B) False color (IR-RedGreen) differentiating and accentuating the red lead pigment, $P L D$ rendering further enhanced with color balance in Adobe Photoshop CC 2015.5; (C) IR image visualizing additions/corrections in different ink; (D) Exaggerated

Shaded image with measurements of the parchment surface relief's section at multiple locations (@) Codex Eyckensis Project \& KU Leuven; for high quality version consult DOI: 10.5281/zenodo.1172646)

Producing 2D+ multi-light reflectance data - whether or not in combination with multi-spectral imaging - for the documentation and study of parchment \& paper media is well recognized as a best-practice approach [15][16]. With the PLD system these acknowledged advantages can be easily acquired and calculated thanks to its automated and streamlined processes. 


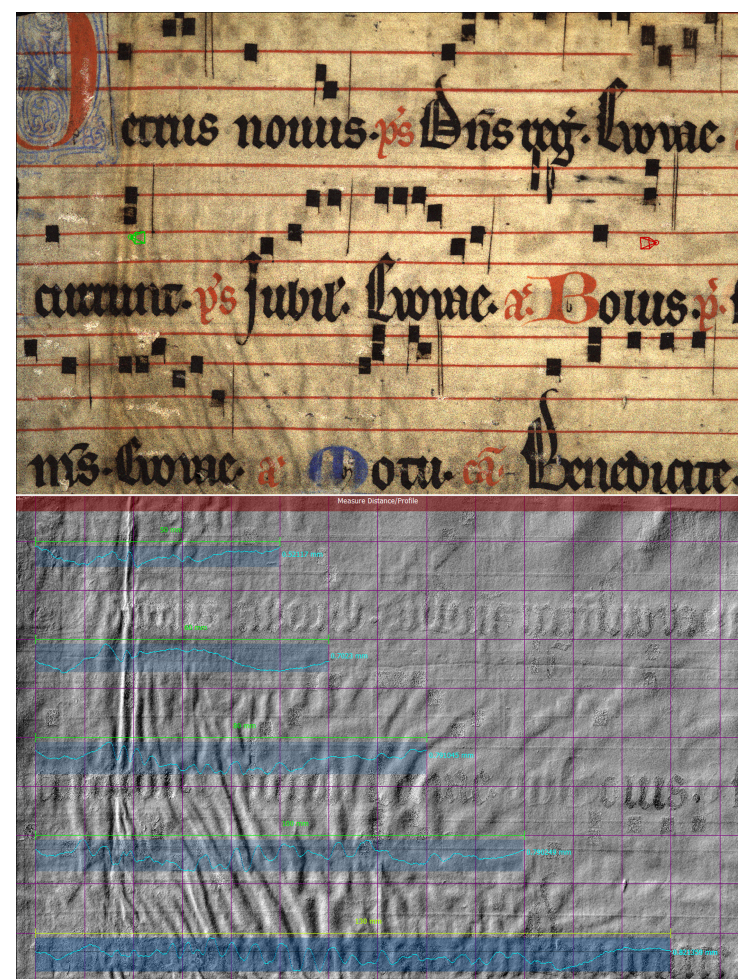

Figure 3. Detail on a $13^{\text {th }}$ century Antiphonarium, parchment (fragment Ms. 1684, Special Collections, KU Leuven Libraries): Monitoring the condition of the document's medium. Renderings based on one recording with the WL PLD system - Above: Standard color representations, the red and green indicators evidence the position of the virtual incident light sources (i.e. albedo); Below: Exaggerated Shaded image with profile measurements of the parchment surface relief's section at multiple locations; in the PLDviewer 7.0.04 a grid $1 \times 1 \mathrm{~cm}$ was activated as visual aid for the monitoring (@ $\mathrm{KU}$ Leuven; for high quality version consult DOI: 10.5281/zenodo.1172974)

A. For the monitoring of the characteristics of the document's medium a 'measuring distance and profile' tool was introduced. In combination with the visual enhancement shaders in the viewer interface (Figure 2D \& 3) it allows conservators to closely observe and value the surface conditions of the imaged object. As a visual aid a grid can be activated with any preferred setting. The tool (i.e. a depth profile) measures any distance in $\mathrm{mm}$ between pixels on the surface in a straight line (x,y-axis, in green \& yellow) and visualizes at the same time the relief of the surface along that stretch in a profile line (z-axis, blue). It derives that information from the data set's normal map, calculated by the photometric stereo processing algorithm. In the case of figure 3 (bottom), the surface was virtually relighted with raking light, emphasizing the undulation folds in the parchment; with the incorporated measuring tool the entire surface can be monitored. Among many applications this allows accurate pre- and post- documentations of conservation treatments. The metric data of the curves can be exported as CSV or text data to support further analysis.

B. The MS module of the PLD system has already been deployed successfully in number of cases for the differentiation and accentuation of particular materials applied on parchment and paper [8][9]. The case presented in Figures 2 and 4 demonstrates several aspects of these abilities. This parchment folio of the 8th century Codex Eyckensis comprises a range of different inks. The start of the gospel in the middle of the folio was written in a red ink containing lead, which has faded extensively and makes it barely legible when standard high-end photography is used (Figure 2A). An automated false color rendering in the PLDviewer software of the multi-spectral data set - the result further on slightly adjusted with a photo editor - is able to accentuate these faded inks and differentiate them with all other features on the imaged surface (Figure 2B). Another interesting feature, which can be observed in detail, are the applied corrections or addenda in the main text, both written in black ink (iron-gall). Especially the normal map of the IR rendering (excitation source $850 \mathrm{~nm}$ ) differentiates the two writing actions distinctly (Figure $2 \mathrm{C} \&$ Figure 4B). With this same data set the characteristics of the inks can even be examined more closely.

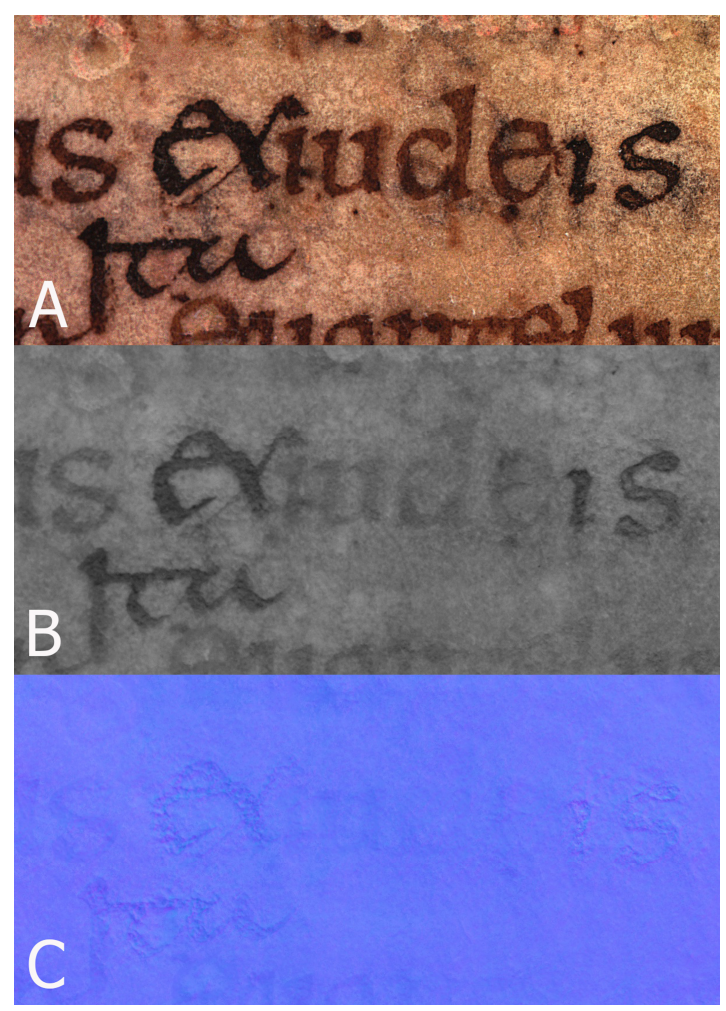

Figure 4. Detail 02 on folio 19v of the 8th century Codex Eyckensis, parchment (@ Musea Maaseik) (see also Figure 2C). Renderings based on one and the same 5 min recording with the MS PLD system - (A) RGB rendering already hinting the use of two different inks; (B) Raw IR image differentiating the two applied inks on the parchment; (C) Normal map based on the Red data set revealing how the surface orientations (relief) of the added ink is distinctively irregular when compared with the dimensional remnants of the main scripture () Codex Eyckensis Project \& KU Leuven; for high quality version consult DOI: 10.5281/zenodo.1172656)

The abilities of the multi-light reflectance's photometric stereo computations reveal as well the surface orientations of each pixel. In Figure 4C it can be observed the relief of the retrofitted characters is irregular, whereas the main text and the relief of the parchment is smooth and flat. The physical topography of both presumable differs as the ink of the retrofitted characters is more dense and lies on top of the parchment. 


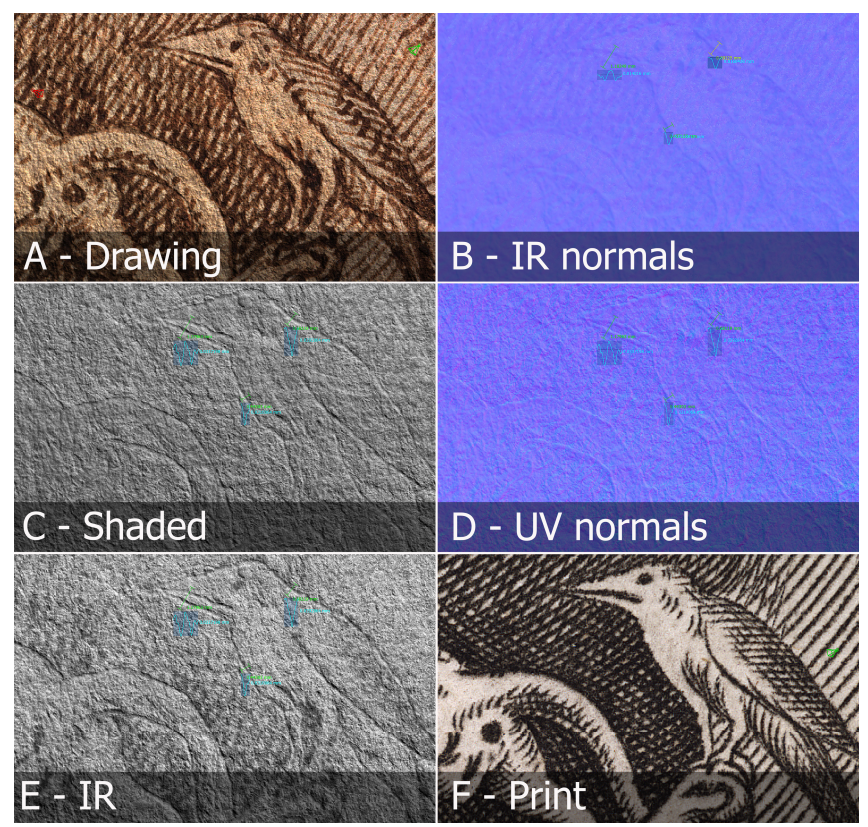

Figure 5. Detail on NHD27 Luxuria by Bruegel, paper (drawing SII132816 \& print SII22656, both (C) KBR, Brussels). The drawing (A-E) has been imaged with the MS PLD; the print (F) with the WL PLD. (A) Standard color representations of the detail on the drawing, the red and green indicators evidence the position of the virtual incident light sources (i.e. albedo); (B) Normal map based on the IR dataset, the indentations are slightly recognized the blue profile lines of the surface reveal the estimated depth of the indentation lines; (C) Surface with texture map, revealing all indentation lines, the underlying normal map is based on the UV dataset; (D) Normal map based on the UV dataset, all indentation lines are accurately recognized by the system; (E) IR texture map with underlying UV dataset normal map, with sufficient raking light an excellent representation of the indentation lines can be achieved; (F) Same zone on a print (mirrored), revealing some slight shifts of the bird's contour lines compared with the indentations on the drawing. (@) Fingerprint Project \& KU Leuven; for high quality version consult DOI: 10.5281/zenodo.1172662)

C. Shallow production and manipulation marks in surfaces have been exposed and visualized with success as well. Whether these are blind ruling lines or even tracing lines on parchment or paper, the photometric stereo algorithm can recognize them in the surfaces relief. An excellent example of this ability arises from an ongoing research on Bruegel the Elder drawings [17]. For one approach the manner in which the master applied the inks has been spectrally unraveled with the MS PLD recordings. But, these same datasets also provided insight on how Bruegel's design on paper was transferred onto copper plates through tracing the drawn lines with a stylus (Figure 5). On the 2D+ images of the original drawing these tracing lines could be visualized or by means of the normal map, the shading filter, or by bringing the results of the IR data set; the two latter in combination with sufficient virtual raking light. Once these indentations were visually localized they can even be metrically analyzed (Figure 5B-E). It permits researchers to assess how the engraver transferred the composition from drawing to copper plate (Figure 5A\&F). Remark also the difference in depth profiles obtained with the IR vs. the UV data sets (Figure 5B\&D). The incised lines based on the UV spectral bands have been estimated more finely.

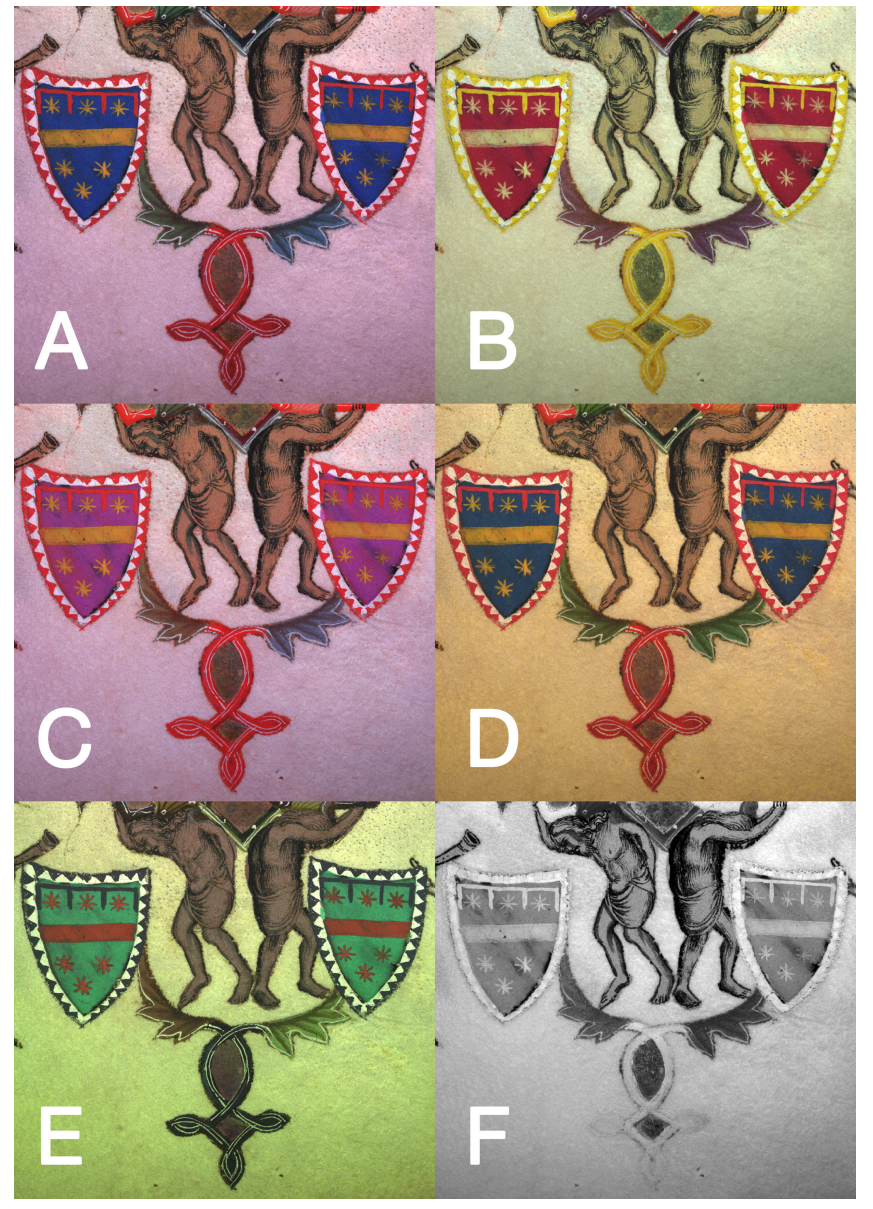

Figure 6. Detail of the coat of arms on folio 230r of the Bible of Anjou (MS1, Maurits Sabbe Library, KU Leuven Libraries). (A-E) showing the automatic created (false) color images in the viewer application: (A): R-G-B. (B) IR-R-G, (C) IR-G-B, (D) R-G-UV, (E) G-B-UV. For the on (A) blue fields, the false color images $(\boldsymbol{B})$ and $(\boldsymbol{C})$ have the same spectral response as the ultra-marine zones on folio 003V; the same goes for the MS histogram of these zones (F) shows the pure IR data, hinting at the overpainted original coat of arms. (C) RICH Project \& KU Leuven; for high quality version consult DOI: 10.5281/zenodo.1186670)

D. Within a number of case studies operating the MS PLD system has allowed the identification of particular types of media (mosaic \& pure gold, azurite, ultra-marine [9]). As there is an even spread of the five different spectral LED's emitters on the dome's inside, the same interactivity as in the WL PLD, such as the virtual relighting and filters, is guaranteed. In addition to that not only the pure results of these five spectra can be assessed individually, (false) color images are produced automatically within the viewer software without the need of an external image editor. As such RG-B, IR-R-G, IR-G-B, R-G-UV and G-B-UV combinations are accessible at the click of a button. This has been proven useful in the assessment of polychrome media. Good case studies for this approach are e.g. illuminated manuscripts, such as folio 230r in the $14^{\text {th }}$ century Bible of Anjou. Earlier research [9] has indicated the use of two different blue pigments on folio $3 \mathrm{v}$ of that same manuscript: azurite and ultra-marine. Through the automated acquisition and processing of the MS PLD system the assessment of the coat of arms on folio 230r, again the use of ultra-marine can be identified, as both visual and metric data (multi-spectral 
histogram) are close to identical of the ultra-marine zones on folio $3 v$ (Figure $6 \& 7$ ). Additionally, the IR data hints the original coat of arms was overpainted (two diagonal bands can be noticed under the blue field), allowing further analysis of the provenance (Figure $6 \mathrm{E})$. The cross comparison between folio $3 \mathrm{v}$ and $230 \mathrm{r}$ is based on two separate MS PLD recordings with the same recording conditions and settings.

Both spectral and (false) color images can be combined with the topographical normal map metric data of the five individual spectra, allowing combinations such as IR spectral image and UV metric data. This has proven very useful.

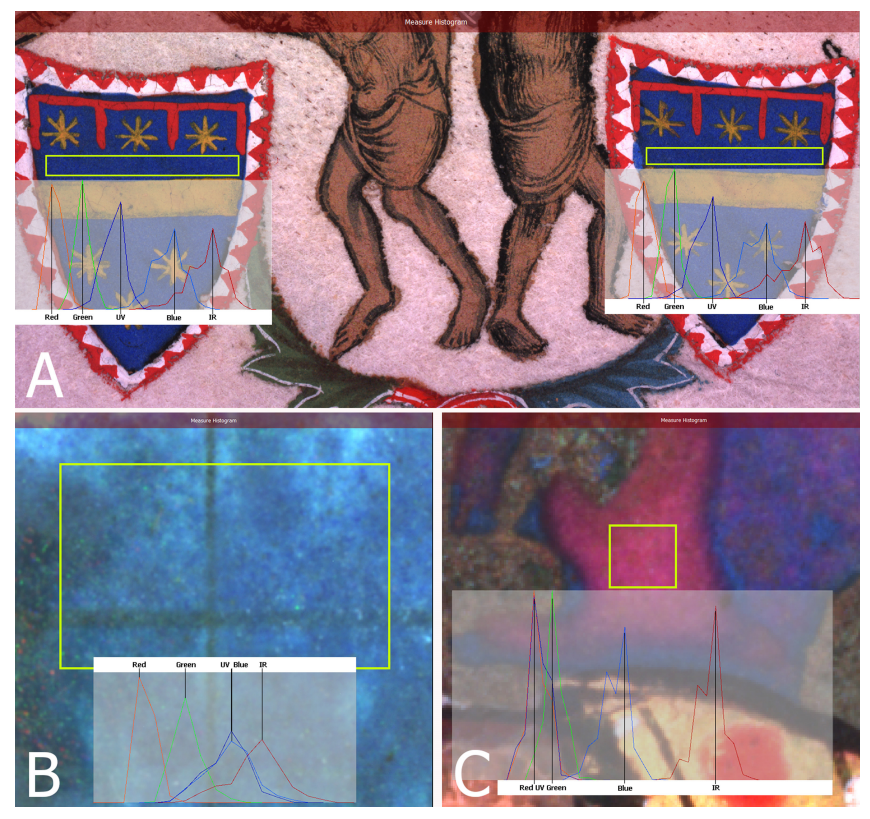

Figure 7. Details on folios $3 v(\boldsymbol{C})$ and 230r (A) of the Anjou Bible (MS1, Maurits Sabbe Library, KU Leuven Libraries) \& of the Ultramarine nat. patch (B) on the Pigments Checker by CHSOS (v.2.1). (A) On both coat of arms a similar blue appearing zone was selected (yellow frame) for which a histogram for the five applied spectra is calculated; image is a $R-G-B$ rendering. (B) MS histogram of the ultramarine pigment, image is a R-G-B rendering. (C) Histogram of a zone with predominantly an ultramarine pigment but partly mixed with azurite, image is an IR-R-G rendering, which differentiates the visual blue appearing ultramarine and azurite in purple and blue respectively. (A-C) The histogram data (all rendered with the same settings in PLDviewer 7.0.05) fuels the conclusion the blue pigment in both coat of arms of folio 230r can be identified as ultramarine (@ RICH Project \& KU Leuven; for high quality version consult DOI: 10.5281/zenodo.1186674)

These are only a few examples of many possible usages for this technology. Further interesting results have been reached, e.g. the study of wax and paper seals, watermarks, decaying parchment, in order to visualize and understand the complex history of origin of objects, differentiating pictorial and pigment layers on illuminated manuscripts, tracing the relief of book bindings, ... [5] [7].

\section{Conclusions}

Tools such as the WL and MS Microdome allow nondestructive analysis of often fragile heritage objects. Combining both visual and analytical tools in one recording session and one output interface is a strong asset. By including all stakeholders into each intermediate step, each of them is made aware of the various demands, technological restrictions and dissemination requests \& opportunities. Successfully bridging multi-light and multi-spectral images provides new opportunities for the elaboration of the strategies for digitizing archives.

\section{References}

[1] G. Willems, F. Verbiest, W. Moreau, H. Hameeuw, K. Van Lerberghe, L. Van Gool, L., "Easy and cost-effective cuneiform digitizing", in The 6th International Symposium on Virtual Reality, Archaeology and Cultural Heritage (VAST 2005). International Symposium on Virtual Reality, Archaeology and Cultural Heritage. Pisa, 2005. Aire-la-Ville: Eurographics Assoc, 73-80, 2005.

[2] H. Hameeuw, G. Willems, "New Visualization Techniques for Cuneiform Texts and Sealings," Akkadica 132/2, 163-178, 2011.

[3] H. Hameeuw, S. van Overmeire, "The Seleucid bullae from Uruk in the Royal Museums of Art and History, Brussels," Mesopotamia. 49, 113-142, pl. 5-13, 2014.

[4] I. Korthagen, F. Prinsen, L. Watteeuw, B. Vandermeulen, "Metamorfoze Digitaliseren van Manuscripten," (version 1.0, July 2016). https://www.metamorfoze.n1/kennisonderzoek/kennisblog/risicos-bij-digitaliseren-vanmanuscripten

[5] L. Watteeuw, B. Vandermeulen, J. Van der Stock, P. Delsaerdt, S. Gradmann, F. Truyen, M. Proesmans, W. Moreau, L. Van Gool, "Imaging Characteristics of Graphic Materials with the Minidome (RICH)," Paper Conservation. Decisions \& Compromise. ICOM-CC graphic documents working group interim meeting. Vienna, Austria, 140-141, 2013.

[6] https://vimeo.com/175578404

[7] G. Proot, A. Bell: "New Scholarship in Early Modern Binding," Journal of Paper Conservation, 15/1, 32-34, 2014. https://youtu.be/253FGAX9aUo

[8] A. Van der Perre, H. Hameeuw, V. Boschloos, L. Delvaux, M. Proesmans, B. Vandermeulen, L. Van Gool, Watteeuw L., "Towards a combined use of IR, UV and 3D-imaging for the study of small inscribed and illuminated artefacts," in Lights On... Cultural Heritage and Museums!, Porto, 163-192, 2016.

[9] L. Watteeuw, H. Hameeuw, B. Vandermeulen, A. Van der Perre, V. Boschloos, L. Delvaux, M. Proesmans, M. Van Bos, L. Van Gool, "Light, shadows and surface characteristics: the multispectral Portable Light Dome," App. Ph. A Mat. Sc. \& Proc. 122: 976, 1-7, 2016.

[10] G. Nam, M. H. Kim, "Multispectral Photometric Stereo for Acquiring High-Fidelity Surface Normals," IEEE Computer Graphics and Applications 34/6, 57-68, 2014.

[11] T. Takatani, Y. Matsushita, S. Lin, Y. Mukaigawa, Y. Yagi 2013, "Enhanced Photometric Stereo with Multispectral Images," in Proceedings of the International Conference on Machine Vision Applications 13, 343-346, 2013.

[12] E. Kotoula, "Reflectance Transformation Imaging Beyond the Visible: Ultraviolet Reflected and Ultraviolet Induced Visible Fluorescence," in Proceedings of the 43rd Annual Conference on Computer Applications and Quantitative Methods in Archaeology, Oxford, 909-919, 2015. 
[13] A. Cosentino, "Terahertz and Cultural Heritage Science: Examination of Art and Archaeology," Technologies 4(1): 6, 2016

[14] E. Kotoula, G. Earl, "Digital research strategies for ancient papyri: a case study on mounted fragments of the Derveni papyrus," in Proceedings of the 42nd Conference on Computer Applications and Quantitative methods in Archaeology 2014, Paris, 145-154, 2014.

[15] C. Schroer, "Advanced Imaging Tools for Museum and Library Conservation and Research," Bulletin of the American Society for Information Science and Technology, Vol. 38/3, 38-42, 2012.

[16] T. R. Hanneken, "New Technology for Imaging Unreadable Manuscripts and Other Artifacts: Integrated Spectral Reflectance Transformation Imaging (Spectral RTI), " in Ancient Worlds in a Digital Culture, Digital Biblical Studies 1, Leiden, 180-195, 2016.
[17] Joris Van Grieken, Lieve Watteeuw, Bruno Vandermeulen, Marc Proesmans, Maarten Bassens, "Fingerprint, projet de recherche sur Pieter Bruegel l'Ancien: Le lancement d'une étude interdisciplinaire de ses dessins et estampes," Science Connection, 45, 32-36 (2017)

\section{Author Biography}

Bruno Vandermeulen: MA Photography, Imaging specialist, head of Digitisation and Document Delivery, KU Leuven Libraries.

Hendrik Hameeuw: Archaeologist and Assyriologist specialized in technical and scientific imaging, Digitisation and Document Delivery, KU Leuven Libraries.

Lieve Watteeuw: Art-Historian and Conservator specialized in art technical research of graphic materials, head of the Book Heritage Lab, KU Leuven, Part time professor at the Faculty of Theology \& at the Faculty of Arts (Illuminare, Centre for the Study of Medieval Art).

Luc Van Gool: Electro-mechanical Engineer, specialized in computer vision research, full professor at PSI Faculty of Engineering Science, KU Leuven and at the Computer Vision Lab TH in Zurich

Marc Proesmans: Electro-mechanical engineer, $\mathrm{PhD}$ in computer vision, Research expert 2D/3D at PSI Faculty of Engineering Science, KU Leuven and co-mgr. Trace. 